\title{
Development of Fuzzy Logic Control for Indoor Lighting Using LEDs Group
}

\author{
M. Khairudin*¹, S. Riyanto ${ }^{2}$, Z. Mohammed ${ }^{3}$ \\ ${ }^{1,2}$ Department of Electrical Engineering, Universitas Negeri Yogyakarta (UNY), Yogyakarta, Indonesia \\ ${ }^{3}$ Faculty of Electrical Engineering, Universiti Teknologi Malaysia (UTM), Johor, Malaysia \\ ${ }^{*}$ Corresponding author, e-mail:moh_khairudin@uny.ac.id
}

\begin{abstract}
This paperpresents the design and the development of an indoor lighting control based on fuzzy logic controller (FLC). The objective of this study is to demonstrate how the FLC can optimize the lighting based on indoor and outdoor lighting environments. The FLC system uses two inputs with Light Dependent Resistors (LDR) as a sensor at indoor and outdoor lighting environments. The output is Light Emitting Diodes (LEDs) to provide lighting at the room automatically. The Pulse Width Modulation (PWM) is used to adjust the $L E D$ s lighting in the room. FLC has successfully demonstrated performance to control the output of LEDs as needed. If the LDR 1 input as indoor sensor shows dim, it will automatically turn on brightly. The MSE values for simulation and experiment of LDR 1 and LDR 2 were 34.42 and 30.11 respectively. The results of FLC performance in the simulation work are further validated by an experimental work. Experimental results show similarities compared to the simulation results.
\end{abstract}

Keywords: fuzzy logic control, indoor lighting, LED

Copyright () 2018 Universitas Ahmad Dahlan. All rights reserved.

\section{Introduction}

The program to realize the green campus with electrical energy saving measures such as replacing the types of lamps become light emiting diode (LED). The LED type lights are used of to save electrical energy [1]. LEDs consume very little power while the light produced is high as well as having a long life time. Therefore, the development of a spatial lighting control system used the LED by increasing the efficiency of the light time and the use of the lamp based on the natural lighting around, it became no waste of electrical energy. Effective lighting system provides better performance to improve equipment efficiency, safety and productivity [2]. The combination of natural lighting from the natural surroundings and the lighting from LED type lights are controlled by using an intelligent system, it is expected to materialize green rooms. Utilization and consumption of energy for household use have reached up to $40 \%$ [3] and [4].

Paralelly with technology development, the use of energy is always increasing especially electrical energy. In 2013 to 2015 the use of electricity in Indonesia increased by $7.2 \%$, ie from 84.43 to 90.48 Tera Watt Hour (TWh) [5]. One of the items was caused by wasteful use of electricity in lighting room especially at homes. During this time, a lot of switching of lighting system still use a conventional switch to turn on and turn off the lights in the room. Particularly the lights in the room is always lit with lighting. As for during the day, the users will turn off manually the lighting because the sun begins to light, this will make the light in the room directly supplied by sunlight. Several studies have been conducted in a smart lighting system based on sensors [6] and [7]. Arindam et al [8] used daylight to achieve energy saving through chopping off peak deman. Furthermore, a fuzzy logic is used to make decision of the number of light intensity through controlling the number of the room lamps [9]. The result achieved the design of fuzzy logic system integrated with electronics hardware of automated light controller to obtain automatic intensity of the room. In the case of building lighting atrium control, [10] used electric lighting energy and visual performance for an atrium building with high frequency dimming controls.

The phenomenon of high use of electrical energy in Indonesia must be overcame well, otherwise there will be an increase in the use of electrical energy. Energy usage should be in accordance with needs. Frequently there is waste of electricity usage. Furthermore, to obtain saving energy [11] generated LED based lighting system by using pico hydro. To make saving 
energy, [12] analysed a total electric energy demand based on the effect of building occupancy concentration using a fuzzy control system. Otherways, to make a respond of the natural state of daylight, [13] used artificial lighting control based on FLC. Aryanto and Seno [14] studied the control of the compact fluorescent lamp to switch on or off according to the condition of the illuminance in the room using FLC with microcontroller. On the effects of using control schemes, [15] conducted an analysis about these control schemes on lighting system having an unique set of factors to affect their performance in terms of energy comsumption and lighting quality.

The fact is the use of home lights, garden lamps, street lights still use the conventional control. During this time the use of natural light energy in everyday life is still not implemented optimally. With the combination of sunlight harmonious and electronic devices such as lights, the use of energy in the room, home, public places can become more efficient. Meanwhile [16] presented a fuzzy logic based lighting system with fluorescent lamp automation to achieve lighting according to Indonesian National Standard (SNI) to achieve energy savings of $75 \%$.

This paper presents a light intensity room control module using FLC as an effort to reduce the use of electrical energy. This study presents the FLC to make the lighting system be automatically light in the room that will be bright and dim in accordance with the outdoors light (sunlight). The effort is conducted by adjusting the electrical energy requirements requested in everyday life while utilizing the source of unlimited natural light energy. Natural light that has not been widely used in this technology will serve as a system stimulant. The lamp as the source of lighting used in this study is LED. The placement of LDR for detecting the lighting is in two different places. The first is placed in the room to detect the amount of light, while the second is placed outside the room to detect the size of natural light. Several works only used the room lighting for FLC input. This work combines the two conditions namely inside room and outside room lightings were monitored to control the lighting room based FLC. In this study, a simulation and experimental works were presented to validate the result. This is rarely conducted by previous researchers who mostly only conduct FLC design until simulation only. The result shows FLC performance that meets the standards in automatically setting the LED lights, this is indicated by the similar result between the simulation and experimental work.

\section{Research Method}

Figure 1 shows the block diagram of an indoor lighting control system used in this work. The rig consists of three main parts: a group of LEDs, Light Dependent Resistors (LDR) sensors and a lab-scale of a room. The indoor lighting control system design consists of a collection of LEDs as a source of space lighting. The LED as the lighting source in this study amounted to 15 pieces of $5 \mathrm{~mm}$ LEDs and arranged circularly centered on the midpoint to obtain optimal light. As for a lab-scale of the room is constructed in Table 1.

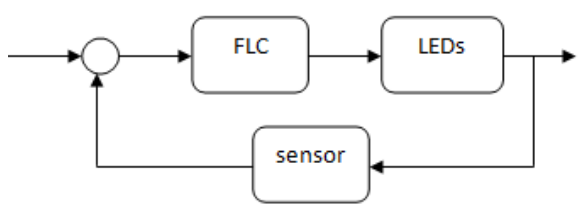

Table 1. Parameters of a lab scale of the room

\begin{tabular}{ccc}
\hline Parameter & Value & Unit \\
\hline Length & 0.50 & $\mathrm{~m}$ \\
Width & 0.50 & $\mathrm{~m}$ \\
Height & 0.40 & $\mathrm{~m}$ \\
Thick of w ood & 0.05 & $\mathrm{~m}$ \\
\hline
\end{tabular}

Figure 1. The block diagram of indoor lighting control

The sensors used in this study were LDR 1 and LDR 2 which were located at the indoor side and the outside respectively. The analogue inputs from both LDR was processed through Analogue to Digital Converter (ADC) that included in microcontroller. Monitoring of system performance was displayed using LCD monitor. Furthermore, the Atmega16 Microcontroller used for data processing in this work was to control a lighting.

Data acquisition and control were accomplished by employing the microcontroller that provided a direct interface between the processor, LEDs and sensors through ADC for analogue input, analogue dan digital output. Two analogue inputs were required in experimental rig for 
input signals of LDR 1 and LDR 2 respectively. The number of 15 LEDs were grouped into three groups, so three digital outputs were used to supply the group of LEDs respectively. The software was developed using CodeVision AVR. To assess the functionality and reliability of lighting control using LDR sensors with FLC algorithm, it is necessary to confirm between natural lighting from the sun and indoor darkness in the room. The FLC will make an efficient controller of an indoor lighting based on outside lighting so that there is no wasting of the lighting.

\section{Results and Analysis}

The automatic control system for indoor lighting used LDR 1 and LDR 2, otherways the LED groups were connected to the ouput respectively. The preparation of FLC consisted of four steps that were determination of fuzzification, dividing in membership function (MF), rule setting and defuzzification.

\subsection{FLC design and simulation}

The determination of fuzzy variable in this study was the light intensity as a result of LDR 1 and LDR 2 input scanning as well as output from LEDs groups. The fuzzy set for LDR 1 and LDR 2 was divided into DARK, DIM, and BRIGHT. While the fuzzy set of LEDs was divided into MINIMAL, MEDIUM, and MAXIMAL. The determination of fuzzification needed to be determined the universe of discourse. The universe of discourse was the total value allowed to be operated in fuzzy variables. The universe of discourse for variables LDR 1 and LDR 2 were $\left[\begin{array}{l}0 \\ 1000\end{array}\right]$ and $[01000]$ respectively, while the universe of discourse for variable LEDs was [0 255]. The fuzzy set domain was the total value allowed in the universe of discourse and might be operated in a fuzzy set. A complete domain set fuzzy can be seen at Table 2.

Next step was the determination of membership function. The membership function (MF) is a curve showing the mapping of data input on a membership value that has an interval between 0 and 1. MF design for input of LDR 1 and LDR 2 can be seen at Figure 2. MF Design of LDR 1 and LDR 2 are arranged with the same parameters because it is possible to have the same characteristics. While the MF design for LEDs groups as ouput in this study is as shown in Figure 3.

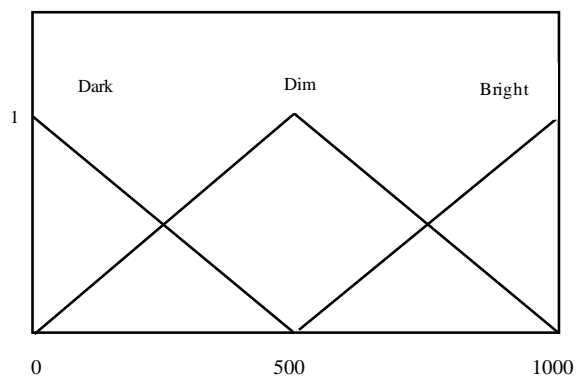

Figure 2. MF design for input of LDR1

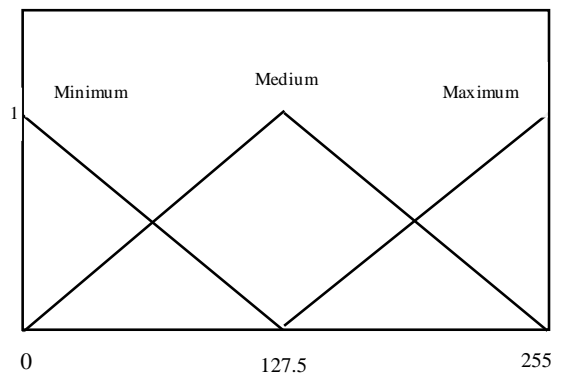

Figure 3. MF design for output of LEDs groups

Table 2. Domain set of input and output fuzzy

\begin{tabular}{cccc}
\hline Variable & Dark & Dim & Bright \\
\hline LDR 1 & {$[-500: 0: 500]$} & {$[0: 500: 1000]$} & {$[500: 1000: 1500]$} \\
LDR 2 & {$[-500: 0: 500]$} & {$[0: 500: 1000]$} & {$[500: 1000: 1500]$} \\
& Minimum & Medium & Maximum \\
LEDs & {$[-127.5: 0: 127.5]$} & {$[0: 127.5: 255]$} & {$[127.5: 255: 382.5]$} \\
\hline
\end{tabular}

After the determination of membership function, the fuzzyfication process was conducted for input and output. In the input type LDR 1, the determination of input variable $(x)$ was the value of ADC for example with value of 100 . The fuzzyfication determination with the Tsukamoto model on the dark MF to get the error value $(\mu)$ was conducted by using the following equation (1): 


$$
\mu D A R K M F=\left\{\begin{array}{cc}
(b-x) /(b-a) ; & a \leq x \leq b \\
0 ; & x \geq b
\end{array}\right.
$$

with $x$ is the value of the LDR sensor 1 input variable from the ADC, thus $b$ and $a$ are upper and lower limits respectively. So for DARK MF when given $x, a$ and $b$ with 100,0 and 500 respectively, then it is obtained $\mu$ dark $=0.8$

The determination of fuzzyfication in this study was done by using Tsukamoto model, so to get the error value $(\mu)$ in DIM MF can be done by using equation (2). With $x$ is the value of the LDR 1 input variable from the ADC, then $c, b$ and $a$ are upper and medium and lower limits respectively. So for DIM MF given $x, a, b$ and $c$ with 100, 0, 500and 1000 respectively, it will be obtained $\mu \mathrm{dim}=0.2$. While the determination of fuzzyfication on MF BRIGHT in order to get the error value $(\mu)$ is by using equation (3).

$$
\begin{gathered}
\mu \text { DIMMF }=\left\{\begin{array}{cr}
0 ; & x \leq a \text { or } x \geq c \\
(x-a) /(b-a) ; & a \leq x \leq b \\
(c-x) /(c-b) ; & b \leq x \leq c
\end{array}\right. \\
\text { н BRIGHTMF }=\left\{\begin{array}{lr}
0 ; & x \leq a \\
(x-a) /(b-a) ; & a \leq x \leq b \\
1 ; & x \geq b
\end{array}\right.
\end{gathered}
$$

With $x$ is the value of LDR sensor 1 input variable from ADC, thus $b$ and $a$ are upper and lower limits respectively. Thus for MF BRIGHT if given $x, a$ and $b$ with 100, 500 and 1000 respectively, then it is obtained $\mu$ bright $=0$. As for the determination of fuzzyfication with Tsukamoto model on LDR 2 with the same equation as in LDR 1 in the process of getting the value of delta_error $(\Delta \mu)$, then it got as in Table 3 .

\begin{tabular}{ccc}
\multicolumn{3}{c}{ Table 3. delta_error $(\Delta \mu)$ value for input of LDR 2 } \\
\hline Classification & Parameters & $\Delta \mu$ \\
\hline Dark & $x=600, a=0, b=500$ & 0 \\
Dim & $x=600, a=0, b=500, c=1000$ & 0.8 \\
Bright & $x=600, a=500, b=1000$ & 0.2 \\
\hline
\end{tabular}

The next step is the determination of the rule. In this study the determination of the rule used AND logic. An $\alpha$ predicate as a result of operation by using AND logic operation was obtained by taking the smallest membership value between elements in the corresponding set according to equation (4).

$$
\mu A \cap B=\min (\mu A(x), \mu B(y))
$$

With $x$ and $y$ are the input variable, thehe determination of the rule by using AND logic can be done with the following equation (5).

\section{IFinpuAconditionANDinput2conditionHENoutputonditior}

So the $\alpha$ predicate value of the rule can be calculated by equation (6) and equation (7).

$$
\begin{aligned}
& \alpha \text { predicate }=\mu L D R \mathrm{1}_{\text {condition }} \cap L D R 2_{\text {condition }} \\
& \alpha \text { predicate }=\min \left(\mu L D R 1_{\text {condition }}(x), \mu L D R 2_{\text {condition }}(x)\right)
\end{aligned}
$$


with $x$ is the value of sensor input variable of LDR 1 and LDR 2. After completing the determination of the rule then the next is the defuzzification process. The defuzzification process can be done by using the following equation (8):

$$
z_{i}=(\alpha \text { predicate } *(b-a))+a
$$

with $b$ and $a$ are upper and lower limits respectively.

The rules and defuzzification process for equation (6), (7) and (8) in this study, where is $\alpha_{\text {pred }_{i}}$ is $\alpha$-predicate, then on R1, R2, and R3 if there are only limits $a$ and $b$ then $a$ and $b$ are lower and upper limits for maximum output respectively, whereas if there is a limit $a, b$ and $c$ then $a, b$ and $c$ are lower, medium and upper limits for maximum output respectively. While at R4 boundary $a, b$ and $c$ are the limit for defuzzification at medium maximum. As for R5 and R6 are for defuzzification on minimal medium. Then R7, R8 and R9 are for defuzzification at minimum output.

After the defuzzyfication process on each rule is completed, next is determining the output value ( $Z$ ) by using Equation (9) follows

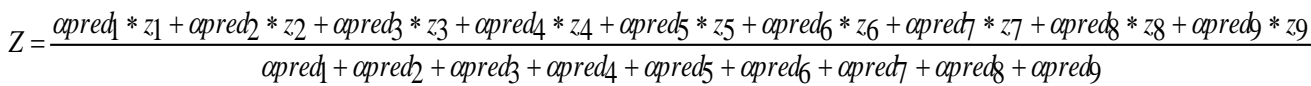

It obtaines the value of output $(Z)$ of 160.29 . This value is used to adjust the size and width of the PWM pulse on the LED light. The simulation process was done using Matlab and Proteus. Each variable in the IF-Then-form rule must be represented by a fuzzy set through a monotonous membership function. As a result, the output of inference results from each rule is given expressly (crisp) based on a-predicate. The final result is obtained by using weighted averages. The process of simulation program testing was done by using Proteus.

The simulation work uses Proteus as shown in Figures 4 and 5. Figure 4 describes a series of Atmega16 microcontroller systems using three ports. PIN A.0 and PIN A.1 are connected to Input of LDR 1 and LDR 2 respectively. Otherways PORT B and PORT C are connected to output of LEDs groups and the LCD display respectively. Based on these simulations, the results obtained were according to the calculations in theory. Thus the program can run well. The results of the simulation can be seen in Tables 4 and 5 .

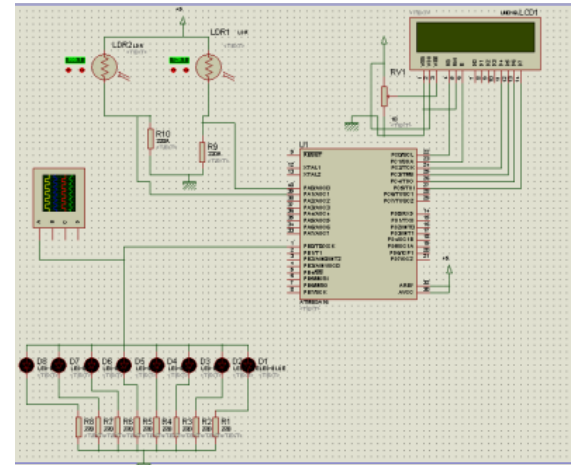

Figure 4. Electrical circuit for simulation

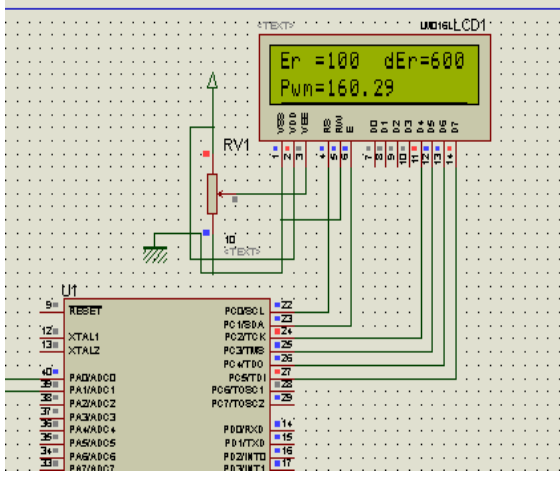

Figure 5. LCD display for error, delta error and PWM

Table 4 shows the results by providing natural light stimulus inside the room in dark conditions up to bright light while the outdoor light is in a relatively stable dim condition. Table 4 presents the FLC has worked with the indication when the LDR 1 input detects light in the dark room (range 0 to 300) and the LDR 2 input detects the outdoor light in the dim range then the LED output will get the maximum supply so it lights up in maximum condition. This condition occurs in the PWM range that controls the LED output between 127.5 to 203.59 . 
While the conditions at which LDR 1 inputs detect light in a dim room (range 300 to 700) and LDR 2 input detects outdoor light in the dim range, then the LED output is in medium condition. This condition occurs in the PWM range that controls the LED output between 93.5 to 127.5. As for the conditions when the LDR 1 input detects light in a bright room (range 700 to 900) and LDR 2 input detects a dimly lit outdoor light, then the LED output is in minimum condition. This condition occurs in the PWM range that controls the LED output between 46.96 to 93.5 .

Table 5 shows the result by providing a natural light stimulus outside the room in dark conditions up to bright light while indoor light is in a relatively stable range of dim conditions. Table 5 presents the FLC has worked with the indication when the LDR 2 input detects dark outdoor light (range 4 to 300) and the LDR 1 input detects light in the dim range room then the LED output will get the medium supply so it lights up in medium condition. This condition occurs in the PWM range that controls the LED output between 126.96 to 127.5. This indicates that the change in external light is not significantly influential, although outside the room shows dark light but if in the room there is still a dim light source then the LEDs will light up in medium condition. In contrast to LDR 1 input which is very influential on LEDs lamp changes, if in the room is identified dark then the LEDs will light up maximally.

Table 4. Simulation results with input of LDR 1.

\begin{tabular}{ccc}
\hline $\begin{array}{c}\text { Inside the Room } \\
\text { (error) }\end{array}$ & $\begin{array}{c}\text { Outside the Room } \\
\text { (Delta error) }\end{array}$ & PWM \\
\hline 0 & 380 & 203.59 \\
100 & 460 & 189.05 \\
200 & 470 & 147.99 \\
300 & 460 & 127.5 \\
400 & 450 & 125.38 \\
500 & 386 & 127.5 \\
600 & 412 & 119.39 \\
700 & 452 & 93.5 \\
800 & 472 & 64.41 \\
900 & 460 & 46.96 \\
\hline
\end{tabular}

Table 5. Simulation results with input of LDR 2

\begin{tabular}{ccc}
\hline $\begin{array}{c}\text { Inside the Room } \\
\text { (error) }\end{array}$ & $\begin{array}{c}\text { Outside the Room } \\
\text { (Delta error) }\end{array}$ & PWM \\
\hline 500 & 4 & 127.5 \\
500 & 100 & 127.5 \\
512 & 200 & 127.36 \\
524 & 300 & 126.96 \\
524 & 400 & 126.96 \\
496 & 500 & 126.5 \\
432 & 600 & 99.13 \\
420 & 700 & 86.08 \\
412 & 800 & 88.08 \\
440 & 900 & 97.56 \\
\hline
\end{tabular}

While the conditions in which LDR 2 inputs detect dimmed outdoor light (range 300 to 700) and LDR 1 input detects indoor light in the dim range, then the LED output is in medium condition. This condition occurs in the PWM range that controls the LED output between 86.08 to 126.5. As the condition when LDR 2 input detects bright outdoor light (range 700 to 900) and LDR 1 input detects indoor light in dim range, then LED output is in minimum condition. This condition occurs in the PWM range that controls the LED output between 86.08 to 97.56. Furthermore, FLC performance of simulation results are validated with experimental results.

\subsection{FLC Implementation and Experimental Results}

Experimental results of the FLC controllers implemented on the experimental indoor lighting are presented in this section. The task of these controllers is to control the lighting of LEDs groups based on the sensors of LDR 1 and LDR 2 located at indoor and outdoor respectively. For real-time implementation of FLC controllers, the code Vision AVR to support compiling and editing the scripts of Atmega16 microcontroller was utilized. This study conducted the experimental control of room lighting with FLC as in the simulation that consisted of 2 inputs in the form of LDR 1 and LDR 2 sensors and the output was divided into 3 groups LEDs. The results of experimental work can be seen in Table 6 and Table 7.

Table 6 presents the performance of FLC with LDR 1 input classification in the fuzzy domain set namely dark, dim and bright. Meanwhile LDR 2 inputs are provided permanently. The output of the FLC system in the form of lighting from the lighting of the LEDs is classified in the fuzzy domain of minimum, medium and maximum with the range of [-127.5: 0: 127.5], [0: 127.5: 255] and [127.5: 255: 382.5] respectively.

Performances of input LDR 1 and LDR 2 and output LEDs are tested by measurement in light intensity (flux) units. Experimental work is conducted by stimulating various LDR 1 input values while LDR 2 is stimulated with a fixed value in the fuzzy domain in the dim range. Table

TELKOMNIKA Vol. 16, No. 3, June 2018: 1165-1173 
6 describes when lighting conditions in the darkened room with indicator low light intensity measured at input LDR 1, then the output will give a high PWM value so that these LEDs will show the higher light intensity. As a result the value of light intensity measurement will be higher. Meanwhile, if there is increase of intensity of light read on the LDR 1, it means that inside the recorded chamber, the illumination becomes brighter then the output will give a low PWM value so that these LEDs will show the lower light intensity. As a result the value of light intensity measurement will be lower. It can be concluded that FLC has shown performance in the classification of input and output. The performance measurement of outdoor light conditions controlled through FLC can be seen in Table 7.

Table 6. Experimental results with of LDR 1

\begin{tabular}{cccc}
\hline $\begin{array}{c}\text { Inside the Room } \\
\text { (Delta) }\end{array}$ & PWM & $\begin{array}{c}\text { Light Intensity of } \\
\text { LDR 1 }\end{array}$ & $\begin{array}{c}\text { Light Intensity of } \\
\text { output }\end{array}$ \\
\hline 0 & 211 & 0 & 2100 \\
100 & 189 & 8 & 1750 \\
200 & 146 & 10 & 1500 \\
300 & 121 & 15 & 1300 \\
400 & 118 & 80 & 1200 \\
500 & 127 & 160 & 1250 \\
600 & 115 & 180 & 1300 \\
700 & 82 & 280 & 850 \\
800 & 65 & 400 & 800 \\
900 & 53 & 1950 & 400 \\
\hline
\end{tabular}

Table 7. Experimental results with of LDR 2

\begin{tabular}{cccc}
\hline $\begin{array}{c}\text { Outside the } \\
\text { Room (Delta) }\end{array}$ & PWM & $\begin{array}{c}\text { Light Intensity } \\
\text { of LDR 2 }\end{array}$ & $\begin{array}{c}\text { Light Intensity } \\
\text { of output }\end{array}$ \\
\hline 4 & 128.4 & 0 & 1300 \\
100 & 127 & 0 & 1300 \\
200 & 127 & 10 & 1300 \\
300 & 127 & 10 & 1300 \\
400 & 127 & 20 & 1300 \\
500 & 122 & 55 & 1200 \\
600 & 92 & 100 & 950 \\
700 & 80 & 420 & 850 \\
800 & 75 & 600 & 850 \\
900 & 93 & 680 & 950 \\
\hline
\end{tabular}

Table 7 presents FLC performance with LDR 2 input classification in the fuzzy domain set namely dark, dim and bright. Meanwhile LDR 1 inputs are provided permanently in the dim range. The output of the FLC system in the form of lighting from the lighting of the LEDs is classified in the fuzzy domain of minimum, medium and maximum with the range of [-127.5: 0 : 127.5], [0: 127.5: 255] and [127.5: 255: 382.5] respectively. The performances of input LDR 1 and LDR 2 and output LEDs are measured in light intensity (flux) units. Experimental testing is done by stimulating various LDR 2 input values while LDR 1 is stimulated with a fixed value in the fuzzy domain dim. Table 7 presents the conditions when the outdoor lighting are darkened by indicators of the low measured light intensity at LDR 2 and the LDR 1 measurement remains in dim condition, then the output will give high PWM value so that these LEDs will show the higher light intensity. As a result the value of light intensity measurement will be higher.

Meanwhile, if the increase of intensity of light read on the LDR 2, it means that outside the recorded chamber illumination, outside the room is brighter, then the output will give a low PWM value so that these LEDs will show the lower light intensity. As a result the value of light intensity measurement will be lower. So it can be concluded that FLC has shown performance in the classification of input and output. It should be noted that external light changes are not significantly influential, although the outdoors show dark light but when in the room there is still a dim light source then the LEDs will light up in medium condition. In contrast to LDR 1 input which is very influential on LEDs lamp changes, if in the room, it is identified dark then the LEDs will light up maximum. This shows the same thing between the simulation and experimental results. 
The result of FLC performance test between simulation and experimental work showed almost the same result and there is only a slight difference. Comparison of FLC performances between simulation and experimental work for input variable of LDR 1 that shows variable of light in the room can be seen in Figure 6. While comparison of FLC performances between simulation and experimental work for input variable LDR 2 or variable of light that exists outside the room is shown in Figure 7.

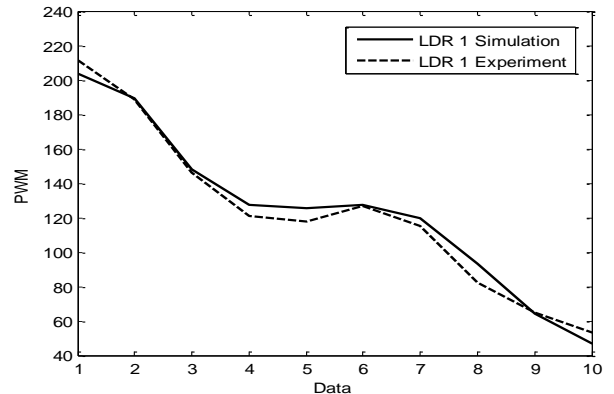

Figure 6. Comparison between simulation and experiment of LDR 1 response

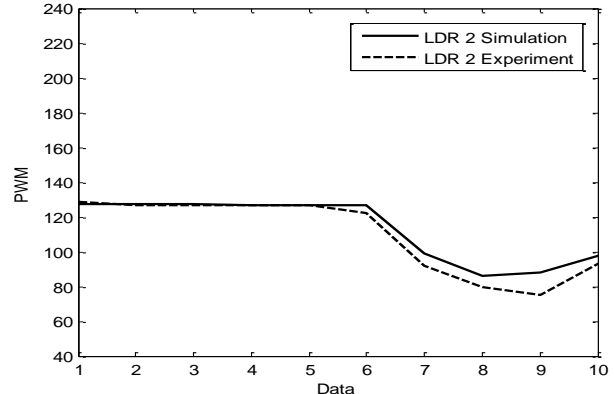

Figure 7. Comparison between simulation and experiment of LDR 2 response

Figures 6 and 7 show the simulation and experimental results have performance similarities, it means that FLC has shown the performance in accordance with the design that has been determined. The comparison between simulation and experimental results is conducted using the Mean Square Error (MSE) method. The MSE values for simulation and experiment of LDR 1 and simulation and experiment of LDR 2 were 34.42 and 30.11 respectively.

\section{Conclusion}

An automatic indoor lighting control system based on FLC is presented. This study features an FLC control system to assess indoor lighting performance with two LDR 1 and LDR 2 for FLC inputs as well as a set of LEDs as outputs. FLC performance assessing has been conducted through simulation and experimental works. The results of a performance assess through simulation shows relatively similar result with experimental work. This shows that the FLC has shown the performance as expected which can set the high and low level intensity of room light based on needs. If indoor sensors and outdoor sensors detect the need to increase the intensity of light, the FLC can meet them as necessary. The results of indoor sensor detection of lighting requirements give a significant impact on FLC performance to control indoor lighting output compared to outdoor lighting changes. This makes it easier for users and streamlines the use of electrical energy.

\section{References}

[1] Moon-Hwan Chang, Peter Sandborn, Michael Pecht, Winco KC Yung, Wenbin Wang. A Return on Investment Analysis of Applying Health Monitoring to LED Lighting Systems. Microelectronics Reliability. 2015; 55(3-4): 527-537.

[2] Yadaf PS, Pal N, Kumar D, Krishna SV. LEDs Lighting Arrangements for Underground Mines. TELKOMNIKA Indonesian Journal of Electrical Engineering. 15.(1): 14-19.

[3] Wen YJ, Granderson J, Agogino AM. Towards embedded wireless-networked intelligent daylighting systems for commercial buildings. Proceeding of IEEE International Conference on Sensor Network Ubiquitous Trustworthy Computing. 2006: 326-331.

[4] L Perez-lombard, J Ortiz, C Pout. A review on buildings energy consumption information. Energy Building. 2008; 40(3): 394-398.

[5] Ministry of Energy and Mineral Resources (Kementerian ESDM). Semester I, Penjualan Tenaga Listrik Tumbuh 7.82\%. Online Bulletin ESDM. Thursday, 14 July 2016. 
[6] Pandharipande A, Caicedo D. Smart Indoor Lighting Systems with Luminaire-based Sensing: A Review of Lighting Control Approaches. Energy \& Buildings. 2015: 369-377.

[7] Kurian CP, Kuriachan S, Bhat J, Aithal RS. An Adaptive Neuro-Fuzzy for the Prediction and Control of Light in Integrated Lighting Schemes. Lighting Research \& Technology. 2015; 37(4): 343-352

[8] Arindam Kumar Sil, Nirmal Kumar Deb, Ashok Kumar Maitra. Energy Saving by Chopping off Peak Demand Using DayLight. TELKOMNIKA (Telecommunication, Computing, Electronics and Control). 2011; 9(2): 395-400.

[9] Kiyanfar M, MahsaLotfibonab, Parisa Lotfi bonab. Automated Room-Light Controller Using Fuzzy Logic. Journal of Artificial Intelligence in Electrical Engineering. 2012; 1(2).

[10] Danny. HWLi, Cheung ACK, Chow SKH, Lee EWM. Study of Daylight Data and Lighting Energy Savings for Atrium Corridor Switch Lighting Dimming Controls. Energy and Buildings. 2014.

[11] Subhashini G, Munandy D, Abdulla R. Generating a Lighting System by Using Pico Hydro System . TELKOMNIKA (Telecommunication, Computing, Electronics and Control). 2017. 15(4): 1565-1573.

[12] Ghadi YY, Rasul MG, Khan MMK. Energy Savings by Fuzzy Base Control of Occupancy Concentration in Institutional Buildings. Energy Procedia. 2017.105: 2850-2858.

[13] Quyen HA, Tan Thanh Tung Le, TrongNghia Le, Thi Minh Thuy Pham. Combining The Daylight and Artificial Light Based on Fuzzy Logic. AETA 2013: Recent Advances in Electrical Engineering and Related Sciences. The Series Lecture Notes in Electrical Engineering. 2013; 282: 93-102.

[14] Hartoyo A, Panjaitan SD. Development of Automation System for Room Lighting Based on Fuzzy logic Controller. International Journal of Information and Electronics Engineering. 2012;2(6).

[15] Toufiq Imam MH, Afshari S, Mishra S. An Experimental Survey of Feedback Control Methodologies for Advanced Lighting Systems. Energy \& Buildings. 2016.

[16] Panjaitan SD, Hartoyo A. A Lighting Control System in Buildings based on Fuzzy Logic. TELKOMNIKA (Telecommunication, Computing, Electronics and Control). 2011; 9(3): 423-432. 